\title{
Similarity and randomness in the molecular clouds associated with Spitzer GLIMPSE Extended Green Objects (EGOs)
}

\author{
J. H. He ${ }^{1}$, S. Takahashi ${ }^{2}$ and X. Chen ${ }^{3}$ \\ ${ }^{1}$ Key Laboratory for the Structure and Evolution of Celestial Objects, Yunnan Astronomical \\ Observatory/National Astronomical Observatory, Chinese Academy of Sciences, P.O. Box 110, \\ Kunming, 650011, Yunnan Province, PR China \\ email: jinhuahe@ynao.ac.cn \\ ${ }^{2}$ Academia Sinica, Institute of Astronomy and Astrophysics, P.O. Box 23-141, Taipei 10617 \\ ${ }^{3}$ Key Laboratory for Research in Galaxies and Cosmology, Key Laboratory of Radio \\ Astronomy, Shanghai Astronomical Observatory, Chinese Academy of Sciences, 80 Nandan \\ Road, Shanghai 200030, PR China
}

\begin{abstract}
EGOs are candidates of massive star forming regions that show signatures of outflows. A $1.1 \mathrm{~mm}$ line survey has been performed to 89 EGOs using the AROSMT. Our high detection rates of $\mathrm{H}^{13} \mathrm{CO}^{+} 3-2$ and $\mathrm{SiO} 6-5$ lines support EGOs to be dense clouds harboring outflows.

Ubiquitous line luminosity linear correlations are found among different kinds of tracer lines: dense gas tracer $\mathrm{H}^{13} \mathrm{CO}^{+}$3-2, outflow tracer $\mathrm{SiO} 6-5$, mixed dense gas and outflow tracers $\mathrm{SO}^{3} \Sigma 6_{5}-5_{4}$ and $\mathrm{CH}_{3} \mathrm{OH}$ lines, and relatively lower density gas tracers ${ }^{12} \mathrm{CO},{ }^{13} \mathrm{CO}, \mathrm{C}^{18} \mathrm{O} 1-0$ (see an example in Fig. 1). This can be explained if a universal similarity of density and thermal structures and probably of shock properties among all these EGO clouds are assumed. Furthermore, the outflow shocks are also required to be produced mainly inside of the natal clouds of the YSOs.

The data scatter of the luminosity (and line width) correlations show a clear trend of worsening across larger cloud substructure size scales or toward larger cloud sizes, which demonstrates the growth of randomness in cloud structures and velocity fields. See more details in our paper (He et al. 2012).
\end{abstract}

Keywords. turbulence — stars: formation — ISM: clouds — ISM: jets and outflows — ISM: kinematics and dynamics — radio lines: ISM

\section{References}

He, J. H., Takahashi, S., \& Chen, X. 2012, ApJS, 202, 1
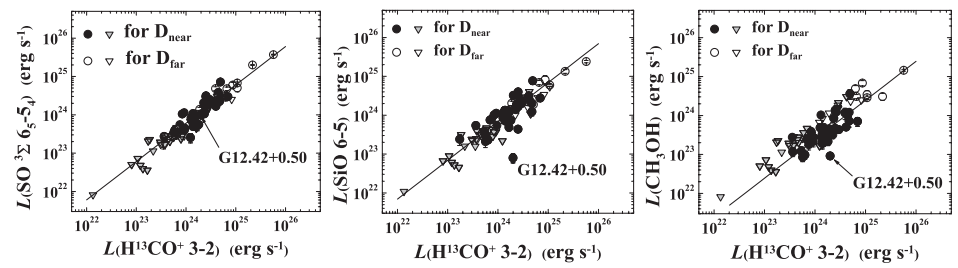

Figure 1. An example of the ubiquitous log-linear correlations among all observed line luminosities: the correlations among the dense gas and shock trackers. Upper limits are shown in triangles, and far distance quantities in open circles. The exceptionally weak $\mathrm{SiO}$ and $\mathrm{CH}_{3} \mathrm{OH}$ lines of $\mathrm{G} 12.42+0.50$ have been excluded from the log-linear fitting (straight lines). All the fitted lines have a slope of unity in log scales and thus also represent linear correlations. 